\title{
GnasisWisdam
}

REVISTA DE INVESTIGACIÓN E INNOVACIÓN CIENTÍFICA Y TECNOLÓGICA

Artículo original

Volumen 1, Número 3, Setiembre - Diciembre 2021

\section{GRADO DE USO DEL PRINCIPIO DE PRIMACÍA DE LA REALIDAD EN LA RESOLUCIÓN DE CONFLICTOS LABORALES EN EL DISTRITO JUDICIAL DE PUNO - PERIODO 2019}

\section{DEGREE OF USE OF THE PRINCIPLE OF PRIMACY OF REALITY IN THE RESOLUTION OF LABOR DISPUTES IN THE JUDICIAL DISTRICT OF PUNO - PERIOD 2019}

\begin{abstract}
Autores
Zarate Pacovilca, Jusely Elma ORCID

Universidad Nacional de Huancavelica
\end{abstract}

\section{Resumen}

Una de las herramientas más relevantes del Derecho del Trabajo es la aplicación de los principios, entre los cuales destaca el denominado principio de primacía de la realidad, de enorme arraigo en la jurisprudencia y los órganos administrativos nacionales. El principio de primacía consiste en que, en caso de discordancia entre lo que ocurre. Una de las herramientas más relevantes del Derecho del Trabajo es la aplicación de los principios, entre los cuales destaca el denominado principio de primacía de la realidad, de enorme arraigo en la jurisprudencia y los órganos administrativos nacionales. El principio de primacía consiste en que, en caso de discordancia entre lo que ocurre en la práctica y lo que surge de documentos o contratos, debe otorgarse preferencia a lo primero, es decir, a lo que sucede y se aprecia en el terreno de los hechos. Así, en virtud de este principio laboral, aun cuando exista un contrato (formalizado por escrito) de naturaleza civil, lo que determina la naturaleza de una relación contractual entre las partes es la forma como en la práctica se ejecuta dicho contrato; es decir la preeminencia de la realidad material sobre lo estipulado en el contrato.

Palabras clave: Realidad, primacía, conflictos laborales.

\begin{abstract}
One of the most important tools of labor law is the application of principles, among which the so-called principle of primacy of reality, huge roots in jurisprudence and national administrative bodies. The principle of primacy is that, in cases of discrepancy between what happens. One of the most important tools of labor law is the application of principles, ñamong which the so-called principle of primacy of reality, huge roots in jurisprudence and national administrative bodies. The principle of primacy is that, in cases of discrepancy between what happens in practice and what emerges from documents or contracts, preference should be given to the first, ie what happens and appreciated in the field of acts. Thus, under this labor principle, even if there is a contract (formalized in writing) of civil nature, which determines the nature of a contractual relationship between the parties it is how in practice the contract is executed; is the preeminence of material reality on the terms of the contract.
\end{abstract}

Keywords: Reality, primacy, labor conflicts.

\section{Introducción}

Plano de la organización productiva y del trabajo, el derecho civil en ese entonces con su ya conocida libertad e igualdad proclamada generaba una relación poco congruente llevada al derecho del trabajo. Básicamente porque en esta se entiende que las partes contratantes (trabajador y empleador) son iguales en el 


\section{GnasisWisdam}

plano factico, no obstante, dicha situación no se corroboraba en una real relación laboral.

La revolución industrial generó mayor demanda de mano de obra, es decir aglutinó en los centros de trabajo a muchos trabajadores para el desempeño de labores de acuerdo al giro de la industria, por su puesto esta situación de mayor requerimiento de personal en un contexto donde no existía regulación laboral ocasiono que el empleador desprovisto de límites establezca las condiciones del contrato de acuerdo a sus intereses dejando de lado la parte trabajadora.

Esta situación de absoluta libertad a la parte patronal generó condiciones deplorables de trabajo, una muestra de ello es las interminables horas de trabajo, sueldos ínfimos, condiciones de segundad y saludes inexistentes, lo cual, no tardó en hacer esperar la reacción de los trabajadores, que, provistos únicamente de sus deseos comunes por condiciones justas de trabajo, peticionaron al estado su intervención que en ese momento tenía un rol de abstención. El estado al principio tomo un rol represor contra estas agrupaciones, incluso sancionaba con penas privativas de la libertad, no obstante, los trabajadores no cesaron en su lucha, y después de una continua presión generalizada pudieron producir la intervención del estado, el que se materializo a través de los dispositivos laborales, es ahí que nace el derecho del trabajo, que entiende perfectamente la desigual innata de las partes, y en razón de ese entendimiento, es que compensa a través de las normas ese desequilibrio de las partes.

Hoy en día, las condiciones de trabajo han sufrido grandes cambios en pro del trabajador, pero, no se ha logrado erradicar esa cultura de fuga hacia la arbitrariedad que tiene como propósito por parte del empleador, reducir costos laborales, pues, existen puertas abiertas por donde el empresario se escapa del derecho laboral.

Una de las puertas abiertas al escape del derecho laboral es sin lugar a dudas la utilización de contratos de distinta naturaleza a la laboral, es decir la celebración acuerdos que eclipsen el vínculo laboral comúnmente se aplica el contrato de locación en servicios - o también en el plano laboral, a través de la utilización de los contratos sujetos a modalidad en actividades permanentes de la empresa, que sería terreno fértil para el contrato por tiempo indefinido.

La doctrina, jurisprudencia y la legislación se han percatado de esta actitud evasiva del empleador y han establecido que se debe desenmascarar los acuerdos a favor de lo que ha acontecido en la realidad, en razón de la actitud arbitraria del empleador se ha creado un elemento trascendental aplicable en ese tipo de situaciones, el que recibe el nombre de PRINICIPIO DE PRIMACIA DE LA REALIDAD.

En el presente trabajo académico - ensayo -, analizo el porqué de la evasión, los vestigios que deja la relación laboral escondida, aunado a la aplicación del principio de primacía de la realidad, con el objeto de descubrir el vínculo laboral disimulado.

\section{Objetivos}

\section{Objetivo general:}

- Determinar el grado de uso y valoración del principio de primacía de la realidad en los procesos laborales, por parte de los magistrados que conocen de conflictos e incertidumbres de naturaleza laboral en el distrito judicial de Puno, periodo 2019

- Precisar si el desconocimiento del uso y valor del principio de primacía de la realidad en los 


\section{GnosisWisdom}

procesos laborales contraviene el principio a la tutela jurisdiccional efectiva.

- Analizar doctrinaria y legalmente la figura jurídica del principio de primacía de la realidad.

\section{Objetivos específicos:}

- Revisar el auto admisorio, actas de audiencias únicas y sentencias dictadas por los distintos órganos jurisdiccionales del Distrito Judicial de Puno en materia laboral en el año 2019 respecto a la aplicación del principio de primacía de la realidad.

- Analizar respecto a la aplicación objetiva y subjetiva del principio de primacía de la realidad por parte de los órganos jurisdiccionales del Distrito Judicial de Puno en el año 2019 al resolver conflictos e incertidumbres jurídicas en materia laboral.

- Determinar si los magistrados y el personal jurisdiccional del Distrito Judicial de Puno en el año 2019 encargados de resolver conflictos e incertidumbres en materia laboral tenían la especialización en materia laboral.

- Concretizar si el desconocimiento del uso adecuado y valor del principio de primacía de la realidad en la resolución de conflictos laborales contraviene el principio a la tutela jurisdiccional efectiva.

- Determinar e identificar cuáles son las consecuencias que se generan por el desconocimiento y no uso del principio de primacía de la realidad en el Distrito Judicial de Puno.

\section{Comprobación de la hipótesis en el trabajo operacional (resultados)}

Expedientes tramitados por los juzgados mixtos de las provincias de Puno y San Roman

Conforme lo precisado en el capítulo anterior la muestra representativa del universo físico hace un total de 403 procesos de amparo en contra de resoluciones judiciales tramitados durante el año judicial 2011, los que corresponden a los Juzgados Mixtos de las provincias de Puno y San Román; sin embargo, para el desarrollo del presente trabajo de investigación se determinó sólo tomar una muestra representativa, el cual detallo en la forma siguiente:

\section{Gráfico e interpretación de los resultados}

Mediante la utilización de cuadros comparativos, distribución de frecuencias e histogramas, se pasa a graficar e interpretar los resultados obtenidos de los expedientes analizados.

Sin embargo, es bueno tener presente que la interpretación es el hecho de que un contenido material, ya dado e independiente del intérprete, sea "comprendido" o "traducido" a una nueva forma de expresión. Dicho concepto está muy relacionado con la hermenéutica

La condición básica de una interpretación es ser fiel de alguna manera especificada al contenido original del objeto interpretado.

La relación intérprete-interpretación se considera compleja y cada caso responde a muy variadas 


\section{GnasisWisdam}

finalidades, condiciones y situaciones, lo que plantea multitud de cuestiones y problemas.

Los problemas de interpretación se entienden mejor si se especifica el contexto o marco en el que se hace dicha interpretación. Por ejemplo, no existen los mismos problemas en la interpretación de unas observaciones científicas, que en la interpretación de algunos aspectos culturales. Dada la variedad de campos en los que aparece la necesidad de interpretación, parece necesario hacer una clasificación de ámbitos fundamentales de interpretación.

\section{Tabla 1.}

Flujo de expedientes analizados.

\begin{tabular}{|c|c|c|c|}
\hline Nro. & EXPEDIENTES ANALIZADOS & TOTAL & $\%$ \\
\hline 01 & Primer Juzgado Mixto de la provincia de Puno & 13 & 8 \\
\hline 02 & Segundo Juzgado Mixto de la provincia de Puno & 9 & 5 \\
\hline 03 & Tercer Juzgado Mixto de la provincia de Puno & 6 & 3 \\
\hline 04 & Primer Juzgado Mixto de la provincia de San Román & 75 & 45 \\
\hline \multirow[t]{2}{*}{05} & Segundo Juzgado Mixto de la provincia de San Román & 66 & 39 \\
\hline & TOTAL, GENERAL & 169 & 100 \\
\hline
\end{tabular}

Fuente: Legajos de los Juzgados Mixtos y revisión del Sistema Integrado Judicial.

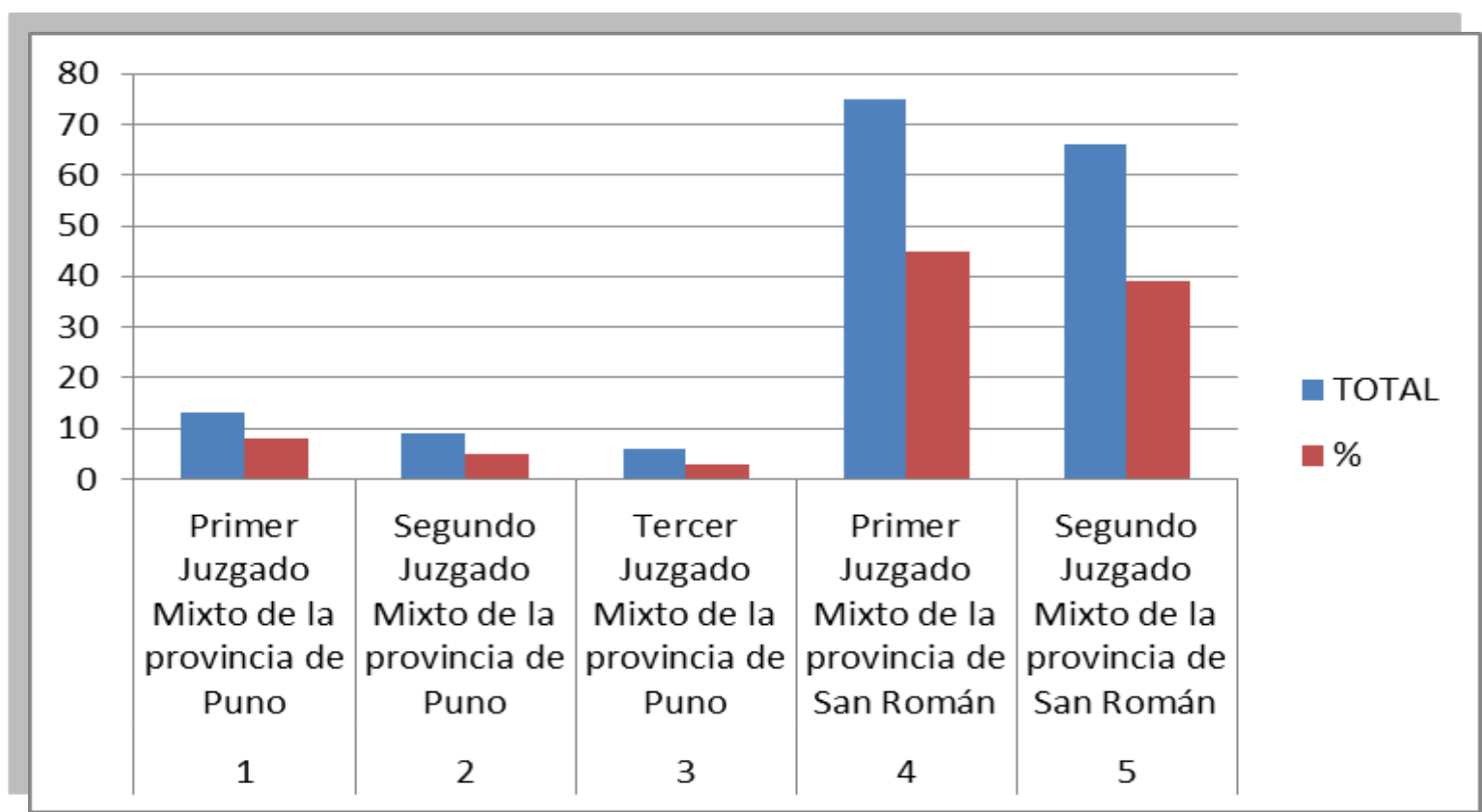

Figura 1

Flujo De Expedientes Analizados. 


\section{GnosisWisdam}

\section{Interpretación}

Del gráfico analizado, se tiene que por ante el Primer Juzgado Mixto de la Provincia de Puno en el año judicial 2011 se tramito un total de 13 procesos constitucionales de acciones de amparo en contra de resoluciones judiciales; por ante el Segundo Juzgado Mixto de la Provincia de Puno en el año judicial 2011 se tramito un total de 9 procesos constitucionales de acciones de amparo en contra de resoluciones judiciales; por ante el Tercer Juzgado Mixto de la Provincia de Puno en el año judicial 2011 se tramito un total de 6 procesos constitucionales de acciones de amparo en contra de resoluciones judiciales; por ante el Primer Juzgado Mixto de la Provincia de San Román en el año judicial 2011 se tramito un total de 75 procesos constitucionales de acciones de amparo en contra de resoluciones judiciales; por ante el Segundo Juzgado Mixto de la Provincia de San Román en el año judicial 2011 se tramito un total de 66 procesos constitucionales de acciones de amparo en contra de resoluciones judiciales; haciendo un total de 169 procesos constitucionales en contra de resoluciones judiciales que se tramitaron por ante los Juzgados Mixtos de las provincias de Puno y San Román, que resulta el $100 \%$ de los procesos revisados y que son materia de estudio.

\section{Referencias}

ABAD YUPANQUI, Samuel B. "El amparo contra leyes" Varios autores - Lecturas constitucionales andinas 3 - Comisión Andina de Juristas - Lima - 1994

ACADEMIA DE LA MAGISTRATURA “El proceso constitucional de amparo" Material de lectura - 2009
BURGOS, Ignacio. "El juicio del amparo" Editorial Themis - Segunda edición - 1950 - Biblioteca de la Universidad Andina "Néstor Cáceres Velásquez" Puno.

BUSTAMANTE DEL CASTILLO, Wilber "Debido proceso y tutela jurisdiccional efectiva" Revista anexa al diario oficial "EL Peruano" Publicación de fecha 04 de marzo de 1999.

CARPIO MARCOS, Edgar "Selección de Jurisprudencias del Tribunal Constitucional" Lima Jurista Editores - 2002

CARRION LUGO, Jorge "Tratado de Derecho Procesal Civil” - Tomo III Lima - Segunda Edición Editora Jurídica GRIJLEY

CARRION LUGO, Jorge "El recurso de casación en el Perú" - Tomo II Lima - Segunda edición - Editorial Jurídica GRIJLEY

CASTAÑEDA OTSU, Susana Ynes, "Derecho Procesal Constitucional" - Tomo II

Lima - Segunda Edición - Editorial Jurídica GRIJLEY

COLAUTTI, Carlos E. "Derechos Humanos” Buenos Aires - Editorial Universidad - 1995.

DIAZ ZEGARRA, Walter, "Código Procesal Constitucional” Lima - Ediciones Legales - 2004

EDITORA NORMAS LEGALES "Jurisprudencia constitucional" Lima - Editora Normas Legales - 2002 GARCIA BELAUNDE, Domingo "El amparo contra resoluciones judiciales de cualquier tipo" Lima Informe profesional - publicada en fecha 28 de Marzo del 2001. 


\section{GnasisWisdam}

JIMENEZ CONDE, F. "Problemas actuales del recurso de amparo" Publicación del anuario de Derecho Constitucional y Parlamentario Nro. 02 - España 1990.

LINARES QUINTANA, Segundo "Tratado de la Ciencia del Constitucional Argentino Comparado" Tomo V - Buenos Aires - Editorial ALFA - 1956.

ORTECHO VILLENA, Víctor Julio "Jurisdicción y Procesos Constitucionales"

Sexta Edición - Lima - Editorial Rodhas 2002.

RANCES "Diccionario Ilustrado de la Lengua Española” España - Editorial SOPENA S.A. - Impreso en España - 1983.

RUBIO CORREA, Marcial "Estudio de la Constitución Política de 1993" VI Tomo - Lima Fondo Editorial de la Pontifica Universidad Católica del Perú - 1999.

SAENZ DAVALOS, Luis R. "Los procesos constitucionales como mecanismos de protección frente a las resoluciones judiciales"

SAENZ DAVALOS, Luis R. "Los procesos constitucionales como mecanismos de protección frente a resoluciones judiciales" Editorial Rodhas Lima - 1999

SAGUES, Néstor Pedro “Acción de amparo", Segunda Edición - Buenos Aires - Editorial ASTREA - 1999.

TICONA POSTIGO "El debido proceso y la demanda civil” II Tomos - Lima - Editorial Rodhas - 1998.

TORRES VASQUEZ, Aníbal "Código Civil” Quinta edición - Lima - Editorial Themis S.A. - 2000 